\title{
Note About Redefinition of BRST Operator for Pure Spinor String in General Background
}

\author{
by J. Klusoň \\ Department of Theoretical Physics and Astrophysics \\ Faculty of Science, Masaryk University \\ Kotlárská 2, 611 37, Brno \\ Czech Republic \\ E-mail: klu@physics.muni.cz
}

\begin{abstract}
We discuss an analysis presented by N. Berkovits in [arXiv:0712.0324] in the context of classical mechanics and perform its extension to the case of pure spinor string in general background.
\end{abstract}

KEYWORDS: string theory. 


\section{Contents}

1. Introduction 1

2. Redefinition of Pure Spinor String BRST Charge in Flat Background 2

3. General Background 10

4. Conclusion 16

5. Appendix: Classical Poisson brackets 17

\section{Introduction}

The pure spinor formalism is a super-Poincare covariant description of the super-string [1] ${ }^{1}$. This new formulation has many attractive properties, for example, it simplifies calculation of multiloop amplitudes [7, 8, 9]. Further it allows to find quantum formulation of superstring in the background with Ramond-Ramond background, at least in principle [10, 11, 12] ${ }^{2}$.

On the other hand due to the fact that the BRST operator in the pure spinor formalism is unconventional the relation of this formalism to the Green-Schwarz (GS) and Ramond-Neveu-Schwarz (RNS) formalisms for the super-string was mysterious ${ }^{3}$. In a recent remarkable paper N. Berkovits [40] explained these features. His idea was to add a pair of non-minimal fields to the theory and perform a similarity transformation such that the pure spinor BRST operator is expressed as a conventional-looking BRST operator that contains collection of first-class constraints. More precisely this conventional-looking BRST operator involves the Virasoro constraints and twelve fermionic constraints, where eleven of these fermionic constraints are associated to the eleven independent components of the original bosonic pure spinor ghost. The additional fermionic constraints and the Virasoro constraints are associated to the new pair of non-minimal fields, bosonic $(\tilde{\beta}, \tilde{\gamma})$ and fermionic $(b, c)$. Even if this conventional form of the BRST operator is not manifestly Lorentz invariant, it was shown that it is useful for construction of $\operatorname{GSO}(-)$ vertex operators and for relating the pure spinor formalism to the GS and RNS formalisms.

Since the analysis presented in [40] was very interesting the goal of this paper is to apply the similar procedure for the pure spinor string in general background. Explicitly, our

\footnotetext{
${ }^{1}$ For review, see [2, 3, 目, 5, 6].

${ }^{2}$ For some related works, see 13, 14, 15, 16, 17, 18, 19, 20, 21, 22, 23].

${ }^{3}$ The problem how pure spinor formalism arises from the conventional GS formalism was attached in many papers from several point of view 24, 25, 26, 28, 29, 29, 30, 31, 32, 33, 34, 35, 36, 37, 38, 39.
} 
starting point is the pure spinor action in general background that was introduced in 441 ${ }^{4}$. As opposite to the original work [41] we formulate the pure spinor action with general worldsheet metric however keeping in mind an important point that pure spinor string is defined on the world-sheet with flat metric. An advantage of this formulation (That of course should be considered as technical tool) is that we can easily find Hamiltonian density as a combination of Virasoro constraints that play a prominent role in Berkovits construction. Then we perform the similarity transformation as in the case of pure spinor string in flat space-time. Since we consider the background as general as possible we do not try to calculate Poisson brackets between $T_{ \pm}$explicitly. Our basic presumption is that the Poisson brackets between $T_{ \pm}$take standard form. Then we argue for an existence of two ghost number -1 functions $G_{ \pm}$that play the role of $b_{ \pm}$ghost fields in the standard formulation. We analyze their Poisson brackets among themselves and with Virasoro constraints. Then we construct operator $R$ and we discuss its basic properties. We show that generally this operator is time dependent and we discuss consequence of its time dependence on the form of the new BRST operator $Q^{\prime}$.

The organization of this paper is as follows. In the next section (2) we review the classical treatment of the pure spinor string in flat Minkowski background. We introduce basic notations and conventions. We also review the approach presented in 440] now formulated in the context of classical mechanics and Poisson brackets.

Then in section (33) we generalize this analysis to the case of pure spinor string in general background. In the first step we develop Hamiltonian formalism for this pure spinor string and find the form of corresponding Hamiltonian and BRST charges. Then we argue for an existence of two functions $G_{ \pm}$that allow to express $T_{ \pm}$as a result of the Poisson brackets of $Q$ with $G_{ \pm}$. Since we consider pure spinor string in general background we will not be able to find explicit form of $G_{ \pm}$and their Poisson brackets with $T_{ \pm}$. On the other hand we can guess the form of these Poisson brackets and try to analyze a consequence of these non-trivial Poisson brackets on the form of the operator $R$. Then we determine the new BRST operator $Q^{\prime}$ that now contains collection of first-class constraints. Namely, if we take pure spinor constraints into account then the new BRST operator $Q^{\prime}$ contains collection of two Virasoro constraints and 22 fermionic constraints. Finally, in conclusion (4) we outline our results and suggest possible extension of this work.

\section{Redefinition of Pure Spinor String BRST Charge in Flat Background}

In this section we review the approach presented in [40]. We perform this analysis in the context of classical Hamiltonian dynamics in order to have a contact with calculation presented in next section.

Our starting point is pure spinor string action in flat background

$$
\begin{aligned}
S & =-\int d^{2} \sigma \sqrt{-h}\left(\frac{1}{2} h^{\mu \nu} \partial_{\mu} x^{m} \partial_{\nu} x^{n} \eta_{m n}+\omega_{\mu \alpha} \mathcal{P}^{\mu \nu} \partial_{\nu} \theta^{\alpha}+\hat{\omega}_{\mu \hat{\alpha}} \tilde{\mathcal{P}}^{\mu \nu} \partial_{\nu} \hat{\theta}^{\hat{\alpha}}+\right. \\
& \left.+w_{\mu \alpha} \mathcal{P}^{\mu \nu} \partial_{\nu} \lambda^{\alpha}+\hat{w}_{\mu \hat{\alpha}} \tilde{\mathcal{P}}^{\mu \nu} \partial_{\nu} \hat{\lambda}^{\hat{\alpha}}\right)
\end{aligned}
$$

\footnotetext{
${ }^{4}$ For discussion of pure spinor string in general background, see $42,43,44,45,46,47$.
} 
where $h^{\mu \nu}$ is two dimensional world sheet metric, $\sigma^{0}=\tau, \sigma^{1}=\sigma$. Further, $\alpha=1, \ldots, 16$ label Majorana-Weyl spinors and $\hat{\alpha}=1, \ldots, 16$ label second Majorana-Weyl spinors and $\omega_{\mu \alpha}, \hat{\omega}_{\mu \hat{\alpha}}$ are related to the momenta conjugate to $\theta^{\alpha}, \hat{\theta}^{\hat{\alpha}} \cdot \gamma_{\alpha \beta}^{m}, \gamma_{\hat{\alpha} \hat{\beta}}^{m}$ are $16 \times 16$ symmetric Dirac matrices. We also introduced chiral and (anti-chiral) operators

$$
\mathcal{P}^{\mu \nu}=h^{\mu \nu}-\frac{\varepsilon^{\mu \nu}}{\sqrt{-h}}, \quad \tilde{\mathcal{P}}^{\mu \nu}=h^{\mu \nu}+\frac{\varepsilon^{\mu \nu}}{\sqrt{-h}},
$$

where $\varepsilon^{\tau \sigma}=-\varepsilon^{\sigma \tau}=1$. Our goal is to develop classical Hamiltonian formalism for pure spinor action (2.1). From (2.1) we determine momenta conjugate to $x^{m}, \theta^{\alpha}, \hat{\theta}^{\hat{\alpha}}$

$$
\begin{aligned}
& p_{m}=\frac{\delta S}{\delta \partial_{\tau} x^{m}}=-\sqrt{-h} h^{\tau \mu} \partial_{\mu} x^{m}, \\
& p_{\alpha}=\frac{\delta S}{\delta \partial_{\tau} \theta^{\alpha}}=\sqrt{-h} \omega_{\mu \alpha} \mathcal{P}^{\mu \tau}, \quad p_{\hat{\alpha}}=\frac{\delta S}{\delta \partial_{\tau} \hat{\theta}^{\hat{\alpha}}}=\sqrt{-h} \hat{\omega}_{\mu \hat{\alpha}} \tilde{\mathcal{P}}^{\mu \tau} .
\end{aligned}
$$

In the same way we proceed in case of pure spinors and we define momenta $\pi_{\alpha}, \hat{\pi}_{\hat{\alpha}}$ conjugate to $\lambda^{\alpha}, \hat{\lambda}^{\hat{\alpha}}$ as

$$
\pi_{\alpha}=\frac{\delta S}{\delta \partial_{\tau} \lambda^{\alpha}}=-\sqrt{-h} w_{\mu \alpha} \mathcal{P}^{\mu \tau}, \quad \hat{\pi}_{\hat{\alpha}}=\frac{\delta S}{\delta \partial_{\tau} \hat{\lambda}^{\hat{\alpha}}}=-\sqrt{-h} \hat{w}_{\mu \hat{\alpha}} \tilde{\mathcal{P}}^{\mu \tau}
$$

However in case of pure spinors there is slight subtlety due to the pure spinor constraints:

$$
\lambda^{\alpha}\left(\gamma^{m}\right)_{\alpha \beta} \lambda^{\beta}=0, \quad \hat{\lambda}^{\hat{\alpha}}\left(\gamma^{m}\right)_{\hat{\alpha} \hat{\beta}} \hat{\lambda}^{\hat{\beta}}=0 .
$$

These relations imply that not all $\lambda$ 's are independent ${ }^{5}$. On the other hand in case of classical calculations presented in this paper we do not have to worry about pure spinor constraints and in all calculations we can tread all $\pi$ 's and $\lambda$ 's as independent. Only in the end of the calculations when we count number of independent constraints we use the explicit parameterization of pure spinors given in footnote.

Let us now return to the review of basic properties of canonical variables. By definition they obey graded Poisson brackets

$$
\begin{aligned}
& \left\{x^{m}(\sigma), p_{n}\left(\sigma^{\prime}\right)\right\}=\delta_{n}^{m} \delta\left(\sigma-\sigma^{\prime}\right), \\
& \left\{\theta^{\alpha}(\sigma), p_{\beta}\left(\sigma^{\prime}\right)\right\}=-\delta_{\beta}^{\alpha} \delta\left(\sigma-\sigma^{\prime}\right), \quad\left\{\hat{\theta}^{\hat{\alpha}}(\sigma), \hat{p}_{\hat{\beta}}\left(\sigma^{\prime}\right)\right\}=-\delta_{\hat{\beta}}^{\hat{\alpha}} \delta\left(\sigma-\sigma^{\prime}\right), \\
& \left\{\lambda^{\alpha}(\sigma), \pi_{\beta}\left(\sigma^{\prime}\right)\right\}=\delta_{\beta}^{\alpha} \delta\left(\sigma-\sigma^{\prime}\right), \quad\left\{\hat{\lambda}^{\hat{\alpha}}(\sigma), \hat{\pi}_{\hat{\beta}}\left(\sigma^{\prime}\right)\right\}=\delta_{\hat{\beta}}^{\hat{\alpha}} \delta\left(\sigma-\sigma^{\prime}\right) .
\end{aligned}
$$

\footnotetext{
${ }^{5}$ We can find set of eleven independent variables when we solve these constraints in $U(5)$ invariant manner. Under $S U(5) \times U(1)$, an $S O(10)$ spinor decomposes as $\lambda^{\alpha} \rightarrow\left(\lambda^{+}, \lambda_{a b}, \lambda^{b}\right)$ where $a=1$ to 5 , $\lambda_{a b}=-\lambda_{b a}$ and $\left(\lambda^{+}, \lambda_{a b}, \lambda^{a}\right)$ carries $U(1)$ charge $\left(\frac{5}{2}, \frac{1}{2},-\frac{3}{2}\right)$. If $\lambda^{+}$is assumed to be nonzero, $\lambda \gamma^{m} \lambda$ implies that

$$
\lambda^{a}=-\frac{1}{8 \lambda^{+}} \epsilon^{a b c d e} \lambda_{b c} \lambda_{d e}
$$

so that $\lambda^{\alpha}$ has eleven independent components parameterized by $\lambda^{+}$and $\lambda_{a b}$.
} 
Further the pure spinor action has to be accompanied with the BRST operators $Q=$ $Q_{L}+Q_{R}$ where two BRST charges take the form

$$
Q_{L}=\int d \sigma \lambda^{\alpha} d_{\alpha}, \quad Q_{R}=\int d \sigma \hat{\lambda}^{\hat{\alpha}} \hat{d}_{\hat{\alpha}}
$$

where

$$
\begin{aligned}
& d_{\alpha}=p_{\alpha}-i p_{m}\left(\gamma^{m} \theta\right)_{\alpha}+\left(\gamma^{n} \theta\right)_{\alpha}\left(\theta \gamma^{m} \partial_{\sigma} \theta\right) \eta_{m n}+i\left(\gamma^{m} \theta\right)_{\alpha} \partial_{\sigma} x^{n} \eta_{m n} \\
& \hat{d}_{\hat{\alpha}}=\hat{p}_{\hat{\alpha}}-i p_{m}\left(\gamma^{m} \hat{\theta}\right)_{\hat{\alpha}}-\left(\gamma^{m} \hat{\theta}\right)_{\hat{\alpha}}\left(\hat{\theta} \gamma^{n} \partial_{\sigma} \theta\right) \eta_{m n}-i\left(\gamma^{m} \hat{\theta}\right)_{\hat{\alpha}} \partial_{\sigma} x^{n} \eta_{m n}
\end{aligned}
$$

We would like to stress that even if we formulated the action (2.1) with the general world-sheet metric $h_{\mu \nu}$ the pure spinor string theory is formulated on the world-sheet with flat world-sheet metric where $h_{\mu \nu}=\eta_{\mu \nu} \equiv \operatorname{diag}(-1,1)^{6}$. Reason why we consider theory with general world-sheet metric is that we can easily develop the Hamiltonian formalism and also find the form of the Virasoro constraints. Explicitly, let us introduce variables

$$
\rho^{ \pm}=\frac{\sqrt{-h} \pm h_{\tau \sigma}}{h_{\sigma \sigma}}, \quad \xi=\ln h_{\sigma \sigma}
$$

where $\rho_{ \pm}$are manifestly invariant under Weyl transformation $h_{\mu \nu}^{\prime}=e^{\phi} h_{\mu \nu}$ while $\xi$ transform as $\xi^{\prime}=\xi+\phi$. Using this notation we can express the projectors (2.2) as

$$
\begin{aligned}
& \mathcal{P}^{\tau \tau}=-4 \frac{e^{-\xi}}{\left(\rho^{+}+\rho^{-}\right)^{2}}, \quad \mathcal{P}^{\sigma \sigma}=4 \frac{\rho^{+} \rho^{-} e^{-\xi}}{\left(\rho^{+}+\rho^{-}\right)^{2}}, \\
& \mathcal{P}^{\tau \sigma}=-4 \frac{\rho^{-} e^{-\xi}}{\left(\rho^{+}+\rho^{-}\right)^{2}}, \quad \mathcal{P}^{\sigma \tau}=4 \frac{\rho^{+} e^{-\xi}}{\left(\rho^{+}+\rho^{-}\right)^{2}}, \\
& \tilde{\mathcal{P}}^{\tau \tau}=4 \frac{e^{-\xi}}{\left(\rho^{+}+\rho^{-}\right)^{2}}, \quad \tilde{\mathcal{P}}^{\sigma \sigma}=4 \frac{\rho^{+} \rho^{-} e^{-\xi}}{\left(\rho^{+}+\rho^{-}\right)^{2}}, \\
& \tilde{\mathcal{P}}^{\tau \sigma}=4 \frac{\rho^{+} e^{-\xi}}{\left(\rho^{+}+\rho^{-}\right)^{2}}, \quad \tilde{\mathcal{P}}^{\sigma \tau}=-4 \frac{\rho^{-} e^{-\xi}}{\left(\rho^{+}+\rho^{-}\right)^{2}} .
\end{aligned}
$$

Then we can easily find the Hamiltonian density in the form

$$
\begin{aligned}
\mathcal{H} & =\partial_{\tau} x^{m} p_{m}+\partial_{\tau} \theta^{\alpha} p_{\alpha}+\partial_{\tau} \hat{\theta}^{\hat{\alpha}} \hat{p}_{\hat{\alpha}}-\mathcal{L}= \\
& =-\frac{1}{\sqrt{-h} h^{\tau \tau}}\left[\frac{1}{2} p_{m} \eta^{m n} p_{n}+\frac{1}{2} \partial_{\sigma} x^{m} \eta_{m n} \partial_{\sigma} x^{n}\right]-\frac{h^{\tau \sigma}}{h^{\tau \tau}} p_{m} \partial_{\sigma} x^{m}- \\
& -\frac{h_{\tau \tau}}{h_{\tau \sigma}+\sqrt{-h}} p_{\alpha} \partial_{\sigma} \theta^{\alpha}-\frac{h_{\tau \tau}}{h_{\tau \sigma}-\sqrt{-h}} \hat{p}_{\hat{\alpha}} \partial_{\sigma} \hat{\theta}^{\hat{\alpha}}+ \\
& +\frac{h_{\tau \tau}}{h_{\tau \sigma}+\sqrt{-h}} \pi_{\alpha} \partial_{\sigma} \lambda^{\alpha}+\frac{h_{\tau \tau}}{h_{\tau \sigma}-\sqrt{-h}} \hat{\pi}_{\hat{\alpha}} \partial_{\sigma} \hat{\lambda}^{\hat{\alpha}}=
\end{aligned}
$$

\footnotetext{
${ }^{6}$ Pure spinor string on world-sheet with general metric was also studied in interesting paper 39.
} 


$$
\begin{aligned}
& =\frac{\rho^{+}+\rho^{-}}{2}\left[\frac{1}{2} p_{m} \eta^{m n} p_{n}+\frac{1}{2} \partial_{\sigma} x^{m} \eta_{m n} \partial_{\sigma} x^{n}\right]+\frac{1}{2}\left(\rho^{+}-\rho^{-}\right) p_{m} \partial_{\sigma} x^{m}+ \\
& +\rho_{-}\left(p_{\alpha} \partial_{\sigma} \theta^{\alpha}-\pi_{\alpha} \partial_{\sigma} \lambda^{\alpha}\right)-\rho_{+}\left(\hat{p}_{\hat{\alpha}} \partial_{\sigma} \hat{\theta}^{\hat{\alpha}}-\hat{\pi}_{\hat{\alpha}} \partial_{\sigma} \hat{\lambda}^{\hat{\alpha}}\right)= \\
& =\rho^{+} T_{+}+\rho^{-} T_{-}
\end{aligned}
$$

where

$$
\begin{aligned}
& T_{-}=\frac{1}{4}\left[p_{m} \eta^{m n} p_{n}+\partial_{\sigma} x^{m} \eta_{m n} \partial_{\sigma} x^{n}\right]-\frac{1}{2} p_{m} \partial_{\sigma} x^{m}+p_{\alpha} \partial_{\sigma} \theta^{\alpha}-\pi_{\alpha} \partial_{\sigma} \lambda^{\alpha}, \\
& T_{+}=\frac{1}{4}\left[p_{m} \eta^{m n} p_{n}+\partial_{\sigma} x^{m} \eta_{m n} \partial_{\sigma} x^{n}\right]+\frac{1}{2} p_{m} \partial_{\sigma} x^{m}-\hat{p}_{\hat{\alpha}} \partial_{\sigma} \hat{\theta}^{\hat{\alpha}}+\hat{\pi}_{\hat{\alpha}} \partial_{\sigma} \hat{\lambda}^{\hat{\alpha}}
\end{aligned}
$$

or alternatively

$$
\begin{aligned}
& T_{-}=\frac{1}{4} \eta^{m n} \Pi_{-m} \Pi_{-n}+d_{\alpha} \partial_{\sigma} \theta^{\alpha}-\pi_{\alpha} \partial_{\sigma} \lambda^{\alpha}, \\
& T_{+}=\frac{1}{4} \eta^{m n} \Pi_{+m} \Pi_{+n}-\hat{d}_{\hat{\alpha}} \partial_{\sigma} \hat{\theta}^{\hat{\alpha}}+\hat{\pi}_{\hat{\alpha}} \partial_{\sigma} \hat{\lambda}^{\hat{\alpha}},
\end{aligned}
$$

where

$$
\begin{aligned}
& \Pi_{+m}=\left(p_{m}+\partial_{\sigma} x^{n} \eta_{n m}-2 i\left(\hat{\theta} \gamma^{n} \partial_{\sigma} \hat{\theta}\right) \eta_{n m}\right), \\
& \Pi_{-m}=\left(p_{m}-\partial_{\sigma} x^{n} \eta_{n m}+2 i\left(\theta \gamma^{n} \partial_{\sigma} \theta\right) \eta_{n m}\right) .
\end{aligned}
$$

Note that we also used following relations

$$
\begin{aligned}
& d_{\alpha \mu} \mathcal{P}^{\mu \sigma}=\rho^{-} d_{\alpha \mu} \mathcal{P}^{\mu \tau}, \\
& \hat{d}_{\hat{\alpha} \mu} \tilde{\mathcal{P}}^{\mu \sigma}=-\rho^{+} d_{\hat{\alpha} \mu} \tilde{\mathcal{P}}^{\mu \tau}
\end{aligned}
$$

that follow from the explicit form of projectors given in (2.11) ${ }^{7}$. Now with the help of the standard Poisson brackets that are collected in Appendix we easily obtain

$$
\begin{aligned}
& \left\{d_{\alpha}(\sigma), T_{-}\left(\sigma^{\prime}\right)\right\}=-d_{\alpha}(\sigma) \partial_{\sigma} \delta\left(\sigma-\sigma^{\prime}\right)-\partial_{\sigma} d_{\alpha}(\sigma) \delta\left(\sigma-\sigma^{\prime}\right), \\
& \left\{\hat{d}_{\hat{\alpha}}(\sigma), T_{+}\left(\sigma^{\prime}\right)\right\}=\hat{d}_{\hat{\alpha}}(\sigma) \partial_{\sigma} \delta\left(\sigma-\sigma^{\prime}\right)+\partial_{\sigma} \hat{d}_{\hat{\alpha}}(\sigma) \delta\left(\sigma-\sigma^{\prime}\right) .
\end{aligned}
$$

Then it is easy to determine the Poisson brackets between $Q$ and $T_{+}, T_{-}$and we obtain

$$
\begin{aligned}
& \left\{Q_{L}, T_{+}(\sigma)\right\}=0, \quad\left\{Q_{R}, T_{+}(\sigma)\right\}=0, \\
& \left\{Q_{L}, T_{-}(\sigma)\right\}=0, \quad\left\{Q_{R}, T_{-}(\sigma)\right\}=0 .
\end{aligned}
$$

\footnotetext{
${ }^{7}$ It is also clear that this relations hold for further chiral variables.
} 
Further, after some algebra we determine the Poisson bracket between Virasoro constraints

$$
\begin{aligned}
& \left\{T_{+}(\sigma), T_{+}\left(\sigma^{\prime}\right)\right\}=2 T_{+}(\sigma) \partial_{\sigma} \delta\left(\sigma-\sigma^{\prime}\right)+\partial_{\sigma} T_{+}(\sigma) \delta\left(\sigma-\sigma^{\prime}\right), \\
& \left\{T_{-}(\sigma), T_{-}\left(\sigma^{\prime}\right)\right\}=-2 T_{-}(\sigma) \partial_{\sigma} \delta\left(\sigma-\sigma^{\prime}\right)-\partial_{\sigma} T_{-}(\sigma) \delta\left(\sigma-\sigma^{\prime}\right), \\
& \left\{T_{+}(\sigma), T_{-}\left(\sigma^{\prime}\right)\right\}=0 .
\end{aligned}
$$

We see that these Poisson brackets take standard form. This fact will be important bellow.

Now we come to an important point in pure spinor formalism. Although there is no fundamental $b$ ghost in the pure spinor formalism one can construct a composite operators $G_{ \pm}$that obey the relations

$$
\left\{Q, G_{+}(\sigma)\right\}=T_{+}(\sigma), \quad\left\{Q, G_{+}(\sigma)\right\}=T_{+}(\sigma)
$$

where

$$
\begin{aligned}
G_{+} & =\frac{\hat{C}_{\hat{\alpha}} G_{+}^{\hat{\alpha}}}{\hat{C}_{\hat{\alpha}} \hat{\lambda}^{\hat{\alpha}}}, \quad G_{-}=\frac{C_{\alpha} G_{-}^{\alpha}}{C_{\alpha} \lambda^{\alpha}}, \\
G_{+}^{\hat{\alpha}} & =-\frac{i}{8} \Pi_{+}^{m}\left(\gamma_{m} \hat{d}\right)^{\hat{\alpha}}+\frac{1}{4} \hat{N}_{m n}\left(\gamma^{m n} \partial_{\sigma} \hat{\theta}\right)^{\hat{\alpha}}+\frac{1}{4} \hat{J} \partial_{\sigma} \hat{\theta}^{\hat{\alpha}} \\
G_{-}^{\alpha} & =-\frac{i}{8} \Pi_{-}^{m}\left(\gamma_{m} d\right)^{\alpha}-\frac{1}{4} N_{m n}\left(\gamma^{m n} \partial_{\sigma} \theta\right)^{\alpha}-\frac{1}{4} J \partial_{\sigma} \theta^{\alpha}
\end{aligned}
$$

Alternatively, using the fact that $\left\{Q, \lambda^{\alpha}\right\}=0$ we can write

$$
\left\{Q, G_{-}\right\}=\frac{C_{\alpha}}{C_{\alpha} \lambda^{\alpha}}\left\{Q, G_{-}^{\alpha}\right\}=T_{-} .
$$

In fact, using Poisson brackets given in Appendix and using important identities

$$
\begin{aligned}
& \delta_{\beta}^{\gamma} \delta_{\alpha}^{\delta}=\frac{1}{2} \gamma_{\alpha \beta}^{m} \gamma_{m}^{\gamma \delta}-\frac{1}{8}\left(\gamma^{m n}\right)_{\alpha}^{\gamma}\left(\gamma_{m n}\right)_{\beta}^{\delta}-\frac{1}{4} \delta_{\alpha}^{\gamma} \delta_{\beta}^{\delta}, \\
& \left(\gamma^{m}\right)_{\gamma \delta}\left(\gamma_{m}\right)_{\alpha \beta}+\left(\gamma^{m}\right)_{\gamma \alpha}\left(\gamma_{m}\right)_{\beta \delta}+\left(\gamma^{m}\right)_{\gamma \beta}\left(\gamma_{m}\right)_{\delta \alpha}=0
\end{aligned}
$$

we can show that the Poisson brackets between $Q$ and $G_{+}^{\alpha}$ given in 2.21) is equal to

$$
\left\{Q, G_{-}^{\alpha}\right\}=\lambda^{\alpha} T_{-}
$$

that confirms (2.22). In the same way we obtain

$$
\left\{Q, G_{+}^{\hat{\alpha}}\right\}=\hat{\lambda}^{\hat{\alpha}} T_{+}
$$

As the next step we calculate the Poisson bracket $\left\{G_{-}(\sigma), G_{-}\left(\sigma^{\prime}\right)\right\}$. The calculation of this bracket is non-trivial and deserves careful calculation. However after some work we derive important result

$$
\left\{G_{-}(\sigma), G_{-}\left(\sigma^{\prime}\right)\right\}=0
$$


It is important to stress that the Poisson bracket given above vanish on condition that $C^{\alpha}$ is pure spinor: $C^{\alpha}\left(\gamma^{m}\right)_{\alpha \beta} C^{\beta}=0$. In the same way we obtain that

$$
\left\{G_{+}(\sigma), G_{+}\left(\sigma^{\prime}\right)\right\}=0 \text {. }
$$

As we will see below the fact that the Poisson brackets (2.26) and (2.27) are zero has an important consequence for the correct redefinition of the BRST operator.

Further we determine the Poisson brackets between $T_{ \pm}$and $G_{ \pm}$. Using the formulas collected in Appendix we easily obtain

$$
\begin{aligned}
& \left\{T_{+}(\sigma), G_{+}\left(\sigma^{\prime}\right)\right\}=-2 G_{+}(\sigma) \partial_{\sigma} \delta\left(\sigma-\sigma^{\prime}\right)-\partial_{\sigma} G_{+}(\sigma) \delta\left(\sigma-\sigma^{\prime}\right), \\
& \left\{T_{-}(\sigma), G_{-}\left(\sigma^{\prime}\right)\right\}=2 G_{-}(\sigma) \partial_{\sigma} \delta\left(\sigma-\sigma^{\prime}\right)+\partial_{\sigma} G_{-}(\sigma) \delta\left(\sigma-\sigma^{\prime}\right) \\
& \left\{T_{ \pm}(\sigma), G_{\mp}\left(\sigma^{\prime}\right)\right\}=0 .
\end{aligned}
$$

After these preliminary calculations we follow [40] and add to the BRST operator (2.8) cohomology trivial term $\int d \sigma\left(\tilde{\gamma}^{+} b_{+}+\tilde{\gamma}^{-} b_{-}\right)$so that the BRST operator takes the form

$$
Q=\int d \sigma\left(\lambda^{\alpha} d_{\alpha}+\hat{\lambda}^{\hat{\alpha}} \hat{d}_{\hat{\alpha}}+\tilde{\gamma}^{+} b_{+}+\tilde{\gamma}^{-} b_{-}\right)
$$

where $\left(\tilde{\beta}_{ \pm}, \tilde{\gamma}^{ \pm}\right)$are bosonic and $\left(b_{ \pm}, c^{ \pm}\right)$are fermionic fields that have following Poisson bracket structure

$$
\left\{c^{ \pm}(\sigma), b_{ \pm}\left(\sigma^{\prime}\right)\right\}=-\delta\left(\sigma-\sigma^{\prime}\right), \quad\left\{\tilde{\gamma}^{ \pm}(\sigma), \tilde{\beta}_{ \pm}\left(\sigma^{\prime}\right)\right\}=\delta\left(\sigma-\sigma^{\prime}\right) .
$$

Using these Poisson bracket and the form of the BRST operator (2.29) we easily determine the transformation properties of these fields under BRST transformations

$$
\begin{aligned}
& \left\{Q, c^{+}(\sigma)\right\}=-\tilde{\gamma}^{+}(\sigma), \quad\left\{Q, c^{-}(\sigma)\right\}=-\tilde{\gamma}^{-}(\sigma), \\
& \left\{Q, \tilde{\beta}_{+}(\sigma)\right\}=b_{+}(\sigma), \quad\left\{Q, \tilde{\beta}_{-}(\sigma)\right\}=b_{-}(\sigma) .
\end{aligned}
$$

We also suggest that these fields contribute to the Hamiltonian density as

$$
\begin{aligned}
\delta H & \equiv \int d \sigma\left(\rho^{+}\left(-\tilde{\beta}_{+} \partial_{\sigma} \tilde{\gamma}^{+}-\partial_{\sigma}\left(\tilde{\beta}_{+} \tilde{\gamma}^{+}\right)+b_{+} \partial_{\sigma} c^{+}\right)+\right. \\
& \left.+\rho^{-}\left(\tilde{\beta}_{-} \partial_{\sigma} \tilde{\gamma}^{-}+\partial_{\sigma}\left(\tilde{\beta}_{-} \tilde{\gamma}^{-}\right)-b_{-} \partial_{\sigma} c^{-}\right)\right)
\end{aligned}
$$

since then the time evolution of $\tilde{\beta}, \tilde{\gamma}, b, c$ has an expected form

$$
\begin{aligned}
& \partial_{\tau} \tilde{\beta}_{ \pm}=\left\{\delta H, \tilde{\beta}_{ \pm}\right\}= \pm \partial_{\sigma}\left(\rho^{ \pm} \tilde{\beta}_{ \pm}\right) \\
& \partial_{\tau} \tilde{\gamma}^{ \pm}=\left\{\delta H, \tilde{\gamma}^{ \pm}\right\}= \pm \rho^{ \pm} \partial_{\sigma} \tilde{\gamma}^{ \pm} \\
& \partial_{\tau} c^{ \pm}=\left\{\delta H, c^{ \pm}\right\}= \pm \rho^{ \pm} \partial_{\sigma} c^{ \pm} \\
& \partial_{\tau} b_{ \pm}=\left\{\delta H, b^{ \pm}\right\}= \pm \partial_{\sigma}\left(\rho^{ \pm} b_{ \pm}\right)
\end{aligned}
$$


In what follows we return to the standard presumption of the pure spinor formalism that the world-sheet metric is flat. Then $\rho^{+}=\rho^{-}=1$ and using (2.28) we easily determine

$$
\partial_{\tau} G_{ \pm}= \pm \partial_{\sigma} G_{ \pm}
$$

Now we are ready to perform the redefinition of the BRST operator (2.29). Let us consider an operator

$$
R=\int d \sigma\left(c^{+} G_{+}+c^{-} G_{-}+c^{+} \partial_{\sigma} c^{+} \beta_{+}-c^{-} \partial_{\sigma} c^{-} \beta_{-}\right) .
$$

Using (2.33) and (2.34) it is easy to see that $R$ is conserved

$$
\partial_{\tau} R=\int d \sigma \partial_{\sigma}(\ldots)=0
$$

where we implicitly presume that the world-sheet modes obey appropriate boundary conditions. Our goal is to perform classical analogue of the redefinition of the BRST operator $Q$ that was performed in [40]. In order to clearly understand of this redefinition in the context of classical mechanics we will be slightly formal and consider either matrix valued functions or quantum mechanics operators $F, Q$ and $R$ in the form

$$
F(x)=e^{x R} Q\left(e^{-x R}\right),
$$

where $x$ is free parameter.

As the next step we make an expansion around the point $x=0$ so that

$$
F(x)=\sum_{n=0}^{\infty} \frac{1}{n !} \frac{d^{n} F}{d^{n} x}(0) x^{n}
$$

and where

$$
\begin{aligned}
\frac{d F}{d x}(0) & =[R, Q], \\
\frac{d^{2} F}{d^{2} x}(0) & =[R,[R, Q]], \\
\ldots & \\
\frac{d^{n} F}{d^{n} x}(0) & =\overbrace{[R, \ldots,[R,[R, Q]]]}^{n} .
\end{aligned}
$$

Then putting $x=1$ and using the fact that $F(x=1)=Q^{\prime}$ we obtain the formal expression for $Q^{\prime}$ in the form

$$
Q^{\prime}=Q+\sum_{n=1}^{n} \frac{1}{n !} \overbrace{[R, \ldots,[R,[R, Q]]]}^{n} .
$$

Using the analogy between Poisson brackets in classical mechanics and commutators in quantum mechanics we propose the classical redefinition of the operator $Q^{\prime}$ in the form

$$
Q^{\prime}=Q+\sum_{n=1}^{n} \frac{1}{n !} \overbrace{\{R, \ldots,\{R,\{R, Q\}\}\}}^{n}
$$


where $\{\ldots\}$ corresponds to graded Poisson bracket. In usual situation the sum above terminates after few steps. Before we proceed to the explicit determination of $Q^{\prime}$ we show that the new operator $Q^{\prime}$ is conserved: $\frac{d}{d \tau} Q^{\prime}=0$. In fact, since $\partial_{\tau} R=0$ we easily obtain that

$$
\frac{d Q^{\prime}}{d \tau}=0
$$

Now we proceed to the explicit calculation of $Q^{\prime}$. To do this we have to calculate the Poisson brackets $\{R, Q\},\{R,\{R, Q\}\}, \ldots$ Firstly, we have

$$
\begin{aligned}
\{R, Q\} & =\int d \sigma\left(c^{+}\left(T_{+}-\tilde{\beta}^{+} \partial_{\sigma} \tilde{\gamma}^{+}-\partial_{\sigma}\left(\tilde{\beta}_{+} \tilde{\gamma}^{+}\right)+b_{+} \partial_{\sigma} c^{+}\right)+\right. \\
& \left.+c^{-}\left(T_{-}+\tilde{\beta}_{-} \partial_{\sigma} \tilde{\gamma}^{-}+\partial_{\sigma}\left(\tilde{\beta}_{-} \tilde{\gamma}^{-}\right)-b_{-} \partial_{\sigma} c^{-}\right)+\tilde{\gamma}^{+} G_{+}+\tilde{\gamma}^{-} G_{-}\right),
\end{aligned}
$$

where we used

$$
\begin{aligned}
& \left\{R, c^{ \pm}\right\}=0, \quad\left\{R, \tilde{\beta}^{ \pm}\right\}=0 \\
& \left\{R, \tilde{\gamma}^{ \pm}\right\}=\mp c^{ \pm} \partial_{\sigma} c^{ \pm} \\
& \left\{R, b^{+}\right\}=G^{+}+\partial_{\sigma} c^{+} \tilde{\beta}_{+}+\partial_{\sigma}\left(c^{+} \tilde{\beta}_{+}\right) \\
& \left\{R, b^{-}\right\}=G^{-}-\partial_{\sigma} c^{-} \tilde{\beta}^{-}-\partial_{\sigma}\left(c^{-} \tilde{\beta}_{-}\right)
\end{aligned}
$$

As the next step we calculate $\{R,\{Q, R\}\}$. In fact, using (2.44) we obtain the result

$$
\{R,\{R, Q\}\}=0 \text {. }
$$

In other words we obtain the BRST operator $Q^{\prime}$ in the form

$$
\begin{aligned}
Q^{\prime} & =\int d \sigma\left[c^{+} \tilde{T}_{+}+c^{-} \tilde{T}_{-}+\lambda^{\alpha} d_{\alpha}+\hat{\lambda}^{\hat{\alpha}} \hat{d}_{\hat{\alpha}}+\right. \\
& \left.+\tilde{\gamma}^{+} G_{+}+\tilde{\gamma}^{-} G_{-}+\tilde{\gamma}^{+} b_{+}+\tilde{\gamma}^{-} b_{-}\right],
\end{aligned}
$$

where

$$
\begin{aligned}
& \tilde{T}_{+}=T_{+}-\tilde{\beta}^{+} \partial_{\sigma} \tilde{\gamma}^{+}-\partial_{\sigma}\left(\tilde{\gamma}^{+} \tilde{\beta}_{+}\right)+b_{+} \partial_{\sigma} c^{+}, \\
& \tilde{T}_{-}=T_{-}+\tilde{\beta}_{-} \partial_{\sigma} \tilde{\gamma}^{-}+\partial_{\sigma}\left(\tilde{\beta}_{-} \tilde{\gamma}^{-}\right)-b_{-} \partial_{\sigma} c^{-} .
\end{aligned}
$$

This is the standard form of the BRST operator for closed superstring when we interpret $\left(\tilde{\gamma}^{+}, \lambda^{+}, \lambda_{a b}, \tilde{\gamma}^{-}, \hat{\lambda}^{+}, \hat{\lambda}_{a b}\right)$ as 24 independent bosonic ghosts together with two sets of Virasoro constraints. It was shown in 40 that this action is closely related to Green-Schwarz superstring. It is also nice to see that the new BRST operator contains Virasoro constraints whose presence was hidden in the original formulation of pure spinor string. 


\section{General Background}

In this section we extend the discussion presented in previous section to the case of pure spinor string in general background ${ }^{8}$. Recall that this action takes the form

$$
\begin{aligned}
S & =-\int d^{2} \sigma \sqrt{-h}\left(\frac{1}{2} h^{\mu \nu} g_{\mu \nu}-\frac{1}{2} \epsilon^{\mu \nu} b_{\mu \nu}+\right. \\
& +P^{\alpha \hat{\beta}} d_{\alpha \mu} \mathcal{P}^{\mu \nu} \hat{d}_{\hat{\beta} \nu}+d_{\alpha \mu} \mathcal{P}^{\mu \nu} \partial_{\nu} Z^{M} E_{M}^{\alpha}(Z)+\hat{d}_{\hat{\alpha} \mu} \tilde{\mathcal{P}}^{\mu \nu} \partial_{\nu} Z^{M} E_{M}^{\hat{\alpha}}(Z)+ \\
& +w_{\mu \beta} \lambda^{\alpha} \mathcal{P}^{\mu \nu} \partial_{\nu} Z^{M} \Omega_{M \alpha}^{\beta}+\hat{w}_{\mu \hat{\beta}} \hat{\lambda}^{\hat{\alpha}} \tilde{\mathcal{P}}^{\mu \nu} \partial_{\nu} Z^{M} \hat{\Omega}_{M \hat{\alpha}}^{\hat{\beta}}+C_{\alpha}^{\beta \hat{\gamma}} \lambda^{\alpha} w_{\beta \mu} \tilde{\mathcal{P}}^{\mu \nu} \hat{d}_{\hat{\gamma} \nu}+ \\
& \left.+\hat{C}_{\hat{\alpha}}^{\hat{\beta} \gamma} \hat{\lambda}^{\hat{\alpha}} \hat{w}_{\hat{\beta} \mu} \mathcal{P}^{\mu \nu} d_{\gamma \nu}+S_{\alpha \hat{\gamma}}^{\beta \hat{\delta}} \lambda^{\alpha} w_{\beta \mu} \mathcal{P}^{\mu \nu} \hat{\lambda}^{\hat{\alpha}} \hat{w}_{\hat{\delta} \nu}\right)+S^{\lambda}+S^{\hat{\lambda}}
\end{aligned}
$$

where

$$
S^{\lambda}=-\int d^{2} \sigma \sqrt{-h} w_{\mu \alpha} \mathcal{P}^{\mu \nu} \partial_{\nu} \lambda^{\alpha}, \quad S^{\hat{\lambda}}=-\int d^{2} \sigma \sqrt{-h} \hat{w}_{\mu \hat{\alpha}} \tilde{\mathcal{P}}^{\mu \nu} \partial_{\nu} \hat{\lambda}^{\hat{\alpha}}
$$

Note also that $g_{\mu \nu}$ and $b_{\mu \nu}$ that appear in (3.1) are defined as

$$
G_{\mu \nu}=\partial_{\mu} Z^{M} E_{M}^{a} \partial_{\nu} Z^{N} E_{N}^{b} \eta_{a b}, \quad b_{\mu \nu}=\partial_{\mu} Z^{M} \partial_{\nu} Z^{N} b_{M N}
$$

and where $M=(m, \mu, \hat{\mu})$ are curved superspace indices, $Z^{M}=\left(x^{m}, \theta^{\mu}, \theta^{\hat{\mu}}\right), A=(a, \alpha, \hat{\alpha})$ are tangent superspace indices, $S^{\lambda}, S^{\hat{\lambda}}$ are the flat actions for the pure spinor variables. Finally $E_{M}^{\alpha}, \quad E_{M}^{\hat{\alpha}}, \quad \Omega_{M \alpha}^{\beta}, \quad \hat{\Omega}_{M \hat{\alpha}}^{\hat{\beta}}, \quad P^{\alpha \hat{\beta}}, \quad C_{\alpha}^{\beta \hat{\gamma}}, \quad \hat{C}_{\hat{\alpha}}^{\hat{\beta} \gamma}, \quad S_{\alpha \hat{\gamma}}^{\beta \hat{\delta}}$ are background spacetime fields. Note also that $d_{\mu \alpha}, \hat{d}_{\mu \hat{\alpha}}$ should be treated as independent variables since $p_{\alpha}, \hat{p}_{\hat{\alpha}}$ do not appear explicitly in the action.

As in the flat space the fundamental object of the pure spinor formalism in the general background is the BRST operator $Q=Q_{L}+Q_{R}$ where

$$
Q_{L}=\int d \sigma \lambda^{\alpha} d_{\alpha \mu} \sqrt{-h} \mathcal{P}^{\mu \tau}, \quad Q_{R}=\int d \sigma \hat{\lambda}^{\hat{\alpha}} \hat{d}_{\hat{\alpha} \mu} \sqrt{-h} \tilde{\mathcal{P}}^{\mu \tau}
$$

Properties of these operators were carefully studied in [41] and we recommend this paper for more details.

In order to use the classical formalism we have to express $d_{\mu \alpha}, \hat{d}_{\mu \hat{\alpha}}$ in terms of the canonical variables of the extended phase space spanned by coordinates $\left(Z^{M}, \lambda^{\alpha}, \hat{\lambda}^{\hat{\alpha}}, P_{M}, \pi_{\alpha}, \hat{\pi}_{\hat{\alpha}}\right)$ where

$$
\begin{aligned}
P_{M} & =\frac{\delta S}{\delta \partial_{\tau} Z^{M}}=-\sqrt{-h} h^{\tau \mu} E_{M}^{a} \eta_{a b} \partial_{\mu} Z^{N} E_{N}^{b}+\partial_{\sigma} Z^{N} b_{M N}+ \\
& +\sqrt{-h}\left[E_{M}^{\alpha} d_{\alpha \mu} \mathcal{P}^{\mu \tau}+E_{M}^{\hat{\alpha}} d_{\hat{\alpha} \mu} \tilde{\mathcal{P}}^{\mu \tau}-\right. \\
& \left.-w_{\mu \beta} \lambda^{\alpha} \mathcal{P}^{\mu \tau} \Omega_{M \alpha}^{\beta}-\hat{w}_{\mu \hat{\beta}} \hat{\lambda}^{\hat{\alpha}} \tilde{\mathcal{P}}^{\mu \tau} \hat{\Omega}_{M \hat{\alpha}}^{\hat{\beta}}\right]
\end{aligned}
$$

\footnotetext{
${ }^{8}$ We omit the Fradkin-Tseytlin term $\int \Phi(Z) r$ where $\Phi$ is dilaton super-field and $r$ is world-sheet curvature.
} 
and

$$
\pi_{\alpha}=\frac{\delta S}{\delta \partial_{\tau} \lambda^{\alpha}}=-w_{\mu \alpha} \sqrt{-h} \mathcal{P}^{\mu \tau}, \quad \hat{\pi}_{\hat{\alpha}}=\frac{\delta S}{\delta \partial_{\tau} \hat{\lambda}^{\hat{\alpha}}}=-\hat{w}_{\mu \hat{\alpha}} \sqrt{-h} \tilde{\mathcal{P}}^{\mu \tau}
$$

By definition these momenta obey the canonical graded Poisson brackets

$$
\begin{aligned}
\left\{Z^{M}(\sigma), P_{N}\left(\sigma^{\prime}\right)\right\} & =(-1)^{|M|} \delta_{N}^{M} \delta\left(\sigma-\sigma^{\prime}\right), \\
\left\{\lambda^{\alpha}(\sigma), \pi_{\beta}\left(\sigma^{\prime}\right)\right\} & =\delta_{\beta}^{\alpha} \delta\left(\sigma-\sigma^{\prime}\right), \quad\left\{\hat{\lambda}^{\hat{\alpha}}(\sigma), \hat{\pi}_{\hat{\beta}}\left(\sigma^{\prime}\right)\right\}=\delta_{\hat{\beta}}^{\hat{\alpha}} \delta\left(\sigma-\sigma^{\prime}\right) .
\end{aligned}
$$

As the next step we express $d_{\alpha \mu}$ as functions of canonical variables. To begin with we use the definition of vielbein

$$
E_{A}^{M} E_{M}^{B}=\delta_{A}^{B}
$$

Then

$$
E_{\alpha}^{M} E_{M}^{b}=0, \quad E_{\alpha}^{M} E_{M}^{\hat{\alpha}}=0
$$

and consequently when we multiply $(3.5)$ with $E_{M}^{\alpha}$ from the left we can express $d_{\alpha \mu} \mathcal{P}^{\mu \tau}, \hat{d}_{\hat{\alpha} \mu} \tilde{\mathcal{P}}^{\mu \tau}$ as functions of canonical variables

$$
\begin{aligned}
& d_{\alpha \mu} \mathcal{P}^{\mu \tau}=\frac{1}{\sqrt{-h}} E_{\alpha}^{M}\left[P_{M}-\partial_{\sigma} Z^{N} b_{M N}-\Omega_{M \gamma}^{\beta} \lambda^{\gamma} \pi_{\beta}-\hat{\Omega}_{M \hat{\alpha}}^{\hat{\beta}} \hat{\lambda}^{\hat{\alpha}} \hat{\pi}_{\hat{\beta}}\right] \equiv \frac{1}{\sqrt{-h}} d_{\alpha}, \\
& \hat{d}_{\mu \hat{\beta}} \tilde{\mathcal{P}}^{\mu \tau}=\frac{1}{\sqrt{-h}} E_{\hat{\alpha}}^{M}\left[P_{M}-\partial_{\sigma} Z^{N} b_{M N}-\Omega_{M \gamma}^{\beta} \lambda^{\gamma} \pi_{\beta}-\hat{\Omega}_{M \hat{\alpha}}^{\hat{\beta}} \hat{\lambda}^{\hat{\alpha}} \hat{\pi}_{\hat{\beta}}\right] \equiv \frac{1}{\sqrt{-h}} \hat{d}_{\hat{\alpha}} .
\end{aligned}
$$

It is also useful to introduce the notation

$$
\begin{aligned}
& \Pi_{\mu}^{a}=\partial_{\mu} Z^{M} E_{M}^{a}, \quad P_{a}=E_{a}^{M} P_{M}, \\
& \Pi_{\tau}^{a}=-\frac{1}{\sqrt{-h} h^{\tau \tau}} \eta^{a b} \hat{P}_{b}-\frac{h^{\tau \sigma}}{h^{\tau \tau}} \Pi_{\sigma}^{a},
\end{aligned}
$$

where

$$
\hat{P}_{A}=E_{A}^{M} P_{M}-E_{A}^{M} \partial_{\sigma} Z^{N} b_{M N}-E_{A}^{M} \Omega_{M \beta}^{\alpha} \pi_{\alpha} \lambda^{\beta}-E_{A}^{M} \hat{\Omega}_{M \hat{\beta}}^{\hat{\alpha}} \hat{\pi}_{\hat{\alpha}} \hat{\lambda}^{\hat{\beta}} .
$$

With this notation and after some work we derive the Hamiltonian density for pure spinor string in general background in the form

$$
\begin{aligned}
\mathcal{H} & =\partial_{\tau} \lambda^{\alpha} \pi_{\alpha}+\partial_{\tau} \hat{\lambda}^{\hat{\alpha}} \hat{\pi}_{\hat{\alpha}}+\partial_{\tau} Z^{M} P_{M}-\mathcal{L}= \\
& =-\frac{1}{2} \sqrt{-h} h^{\tau \tau} \Pi_{\tau}^{a} \Pi_{\tau}^{b} \eta_{a b}+\frac{1}{2} \sqrt{-h} h^{\sigma \sigma} \Pi_{\sigma}^{a} \Pi_{\sigma}^{b} \eta_{a b}+ \\
& +\frac{1}{2} \sqrt{-h}\left[C_{\alpha}^{\beta \hat{\gamma}} \lambda^{\alpha} w_{\beta \mu} \tilde{\mathcal{P}}^{\mu \rho_{1}} h_{\rho_{1} \rho_{2}} \tilde{\mathcal{P}}^{\rho_{2} \nu} \hat{d}_{\hat{\gamma} \nu}+\right. \\
& \left.+\hat{C}_{\hat{\alpha}}^{\hat{\beta} \gamma} \hat{\lambda}^{\hat{\alpha}} \hat{w}_{\hat{\beta} \mu} \mathcal{P}^{\mu \rho_{1}} h_{\rho_{1} \rho_{2}} \mathcal{P}^{\rho_{2} \nu} d_{\gamma \nu}+S_{\alpha \hat{\gamma}}^{\beta \hat{\delta}} \lambda^{\alpha} w_{\beta \mu} \mathcal{P}^{\mu \rho_{1}} h_{\rho_{1} \rho_{2}} \mathcal{P}^{\rho_{2} \nu} \hat{\lambda}^{\hat{\alpha}} \hat{w}_{\hat{\delta} \nu}\right]+
\end{aligned}
$$




$$
\begin{aligned}
& +\sqrt{-h}\left[w_{\mu \alpha} \lambda^{\beta} \mathcal{P}^{\mu \sigma} \partial_{\sigma} Z^{M} \Omega_{M \alpha}^{\beta}++\hat{w}_{\mu \hat{\alpha}} \hat{\lambda}^{\hat{\beta}} \tilde{\mathcal{P}}^{\mu \sigma} \hat{\Omega}_{M \hat{\alpha}}^{\hat{\beta}}+\right. \\
& \left.+d_{\alpha \mu} \mathcal{P}^{\mu \sigma} \Pi_{\sigma}+d_{\hat{\alpha} \mu} \tilde{\mathcal{P}}^{\mu \sigma} \Pi_{\sigma}+P^{\alpha \hat{\beta}} d_{\alpha \mu} \mathcal{P}^{\mu \nu} d_{\hat{\beta} \nu}\right] \\
& +\sqrt{-h} w_{\mu \alpha} \mathcal{P}^{\mu \sigma} \partial_{\sigma} \lambda^{\alpha}+\sqrt{-h} \hat{w}_{\mu \hat{\alpha}} \tilde{\mathcal{P}}^{\mu \sigma} \partial_{\sigma} \hat{\lambda}^{\hat{\beta}} \equiv \rho^{+} T_{+}+\rho^{-} T_{-}
\end{aligned}
$$

where

$$
\begin{aligned}
T_{+} & =\frac{1}{4}\left(\hat{P}_{a}+\eta_{a c} \Pi_{\sigma}^{c}\right) \eta^{a b}\left(\hat{P}_{b}+\eta_{b d} \Pi_{\sigma}^{d}\right)-\hat{d}_{\hat{\alpha}} \Pi_{\sigma}^{\hat{\alpha}}-\frac{1}{2}\left(\pi_{\beta} \lambda^{\alpha} C_{\alpha}^{\beta \hat{\gamma}} \hat{d}_{\hat{\gamma}}+\hat{\pi}_{\hat{\beta}} \hat{\lambda}^{\hat{\alpha}} C_{\hat{\alpha}}^{\hat{\beta} \gamma} d_{\gamma}\right)+ \\
& +\frac{1}{2} S_{\alpha \hat{\gamma}}^{\beta \hat{\delta}} \pi^{\alpha} \lambda_{\beta} \hat{\lambda} \hat{\lambda}^{\hat{\gamma}} \pi_{\hat{\delta}}+\frac{1}{2} P^{\alpha \hat{\beta}} d_{\alpha} d_{\hat{\beta}}+\hat{\pi}_{\hat{\alpha}} \partial_{\sigma} \hat{\lambda}^{\hat{\alpha}} \\
T_{-} & =\frac{1}{4}\left(\hat{P}_{a}-\eta_{a c} \Pi_{\sigma}^{c}\right) \eta^{a b}\left(\hat{P}_{b}-\eta_{b d} \Pi_{\sigma}^{d}\right)+d_{\alpha} \Pi_{\sigma}^{\alpha}+\frac{1}{2}\left(\pi_{\beta} \lambda^{\alpha} C_{\alpha}^{\beta \hat{\gamma}} \hat{d}_{\hat{\gamma}}+\hat{\pi}_{\hat{\beta}} \hat{\lambda}^{\hat{\alpha}} C_{\hat{\alpha}}^{\hat{\beta} \gamma} d_{\gamma}\right)- \\
& -\frac{1}{2} S_{\alpha \hat{\gamma}}^{\beta \hat{\delta}} \pi^{\alpha} \lambda_{\beta} \hat{\lambda} \hat{\lambda}^{\hat{\gamma}} \hat{\pi}_{\hat{\delta}}-\frac{1}{2} P^{\alpha \hat{\beta}} d_{\alpha} d_{\hat{\beta}}-\pi_{\alpha} \partial_{\sigma} \lambda^{\alpha} .
\end{aligned}
$$

Following the logic of previous section it seams to be natural to determine Poisson brackets structure between $T_{ \pm}$'s. However this is very difficult task and the resulting Poisson brackets are not very interesting ${ }^{9}$. Then in order to derive some useful results and predictions we presume that the Poisson brackets of the Virasoro components $T_{ \pm}$take standard form

$$
\begin{aligned}
& \left\{T_{+}(\sigma), T_{+}\left(\sigma^{\prime}\right)\right\}=-2 T_{+}(\sigma) \partial_{\sigma} \delta\left(\sigma-\sigma^{\prime}\right)-\partial_{\sigma} T_{+}(\sigma) \delta\left(\sigma-\sigma^{\prime}\right), \\
& \left\{T_{-}(\sigma), T_{-}\left(\sigma^{\prime}\right)\right\}=2 T_{-}(\sigma) \partial_{\sigma} \delta\left(\sigma-\sigma^{\prime}\right)+\partial_{\sigma} T_{-}(\sigma) \delta\left(\sigma-\sigma^{\prime}\right), \\
& \left\{T_{+}(\sigma), T_{-}\left(\sigma^{\prime}\right)\right\}=0 .
\end{aligned}
$$

Let us again introduce two objects $G_{ \pm}$defined as

$$
\left\{Q, G_{ \pm}(\sigma)\right\}=T_{ \pm}(\sigma)
$$

Then using the nilpotence of $Q:\{Q, Q\}=0$ we obtain

$$
\left\{Q, T_{+}(\sigma)\right\}=0, \quad\left\{Q, T_{-}(\sigma)\right\}=0
$$

As the next step we analyze the Poisson brackets between $T_{ \pm}$and $G_{ \pm}$. Without knowledge of explicit form of $G_{ \pm}$we guess their forms as

$$
\begin{aligned}
& \left\{T_{+}(\sigma), G_{+}\left(\sigma^{\prime}\right)\right\}=-2 G_{+}(\sigma) \partial_{\sigma} \delta\left(\sigma-\sigma^{\prime}\right)-\partial_{\sigma} G_{+}(\sigma) \delta\left(\sigma-\sigma^{\prime}\right) \\
& \left\{T_{-}(\sigma), G_{+}\left(\sigma^{\prime}\right)\right\}=2 G_{-}(\sigma) \partial_{\sigma} \delta\left(\sigma-\sigma^{\prime}\right)+\partial_{\sigma} G_{-}(\sigma) \delta\left(\sigma-\sigma^{\prime}\right) .
\end{aligned}
$$

${ }^{9}$ For careful discussion of this problem in the context of Green-Schwarz superstring in general background see 48 . 
To check that this is correct proposal we calculate the Poisson bracket of $Q$ with left and right side of the first equation in (3.18). The Poisson bracket of left-side with $Q$ gives

$$
\begin{aligned}
& \left\{Q,\left\{T_{+}(\sigma), G_{+}\left(\sigma^{\prime}\right)\right\}\right\}=\left\{T_{+}(\sigma),\left\{Q, G_{+}\left(\sigma^{\prime}\right)\right\}\right\}-\left\{G_{+}\left(\sigma^{\prime}\right),\left\{Q, T_{+}(\sigma)\right\}\right\}= \\
= & \left\{T_{+}(\sigma),\left\{Q, G_{+}\left(\sigma^{\prime}\right)\right\}\right\}=\left\{T_{+}(\sigma), T_{+}\left(\sigma^{\prime}\right)\right\}
\end{aligned}
$$

while the Poisson bracket of $Q$ with right-side of (3.18) gives

$$
\begin{aligned}
& -2\left\{Q, G_{+}(\sigma)\right\} \partial_{\sigma} \delta\left(\sigma-\sigma^{\prime}\right)-\partial_{\sigma}\left\{Q, G_{+}(\sigma)\right\} \delta\left(\sigma-\sigma^{\prime}\right)= \\
= & -2 T_{+}(\sigma) \partial_{\sigma} \delta\left(\sigma-\sigma^{\prime}\right)-T_{+}(\sigma) \delta\left(\sigma-\sigma^{\prime}\right) .
\end{aligned}
$$

Collecting these results we derive the Poisson brackets (3.15). In the same way we can proceed with the second equation in (3.15).

Further, let us consider the Poisson bracket $\left\{G_{+}(\sigma), T_{-}\left(\sigma^{\prime}\right)\right\}$ and apply BRST operator $Q$ on it

$$
\left\{Q,\left\{G_{+}(\sigma), T_{-}\left(\sigma^{\prime}\right)\right\}\right\}=\left\{T_{-}\left(\sigma^{\prime}\right),\left\{Q, G_{+}(\sigma)\right\}\right\}-\left\{G_{+}(\sigma),\left\{Q, T_{-}\left(\sigma^{\prime}\right)\right\}\right\}=0
$$

using the fact that $\left\{T_{+}(\sigma), T_{-}\left(\sigma^{\prime}\right)\right\}=\left\{Q, T_{-}\left(\sigma^{\prime}\right)\right\}=0$. Consequently we generally have

$$
\begin{aligned}
& \left\{T_{-}(\sigma), G_{+}\left(\sigma^{\prime}\right)\right\}=\left\{Q, \Omega_{-+}\left(\sigma, \sigma^{\prime}\right)\right\}, \\
& \left\{T_{+}(\sigma), G_{-}\left(\sigma^{\prime}\right)\right\}=\left\{Q, \Omega_{-+}\left(\sigma, \sigma^{\prime}\right)\right\}
\end{aligned}
$$

for some ghost number -2 functions $\Omega_{-+}$and $\Omega_{+-}$. However the fact that the Poisson bracket between $T_{ \pm}$and $G_{\mp}$ is non-zero has impact on time evolution of $G_{+}$since

$$
\begin{aligned}
& \partial_{\tau} G_{+}=\left\{H, G_{+}\right\}=\partial_{\sigma}\left(\rho^{+} G_{+}\right)+\left\{Q, \boldsymbol{\Omega}_{+-}\right\}, \\
& \partial_{\tau} G_{-}=\left\{H, G_{-}\right\}=-\partial_{\sigma}\left(\rho^{-} G_{-}\right)+\left\{Q, \boldsymbol{\Omega}_{-+}\right\}
\end{aligned}
$$

where

$$
\boldsymbol{\Omega}_{-+}(\sigma)=\int d \sigma^{\prime} \Omega_{-+}\left(\sigma, \sigma^{\prime}\right), \quad \boldsymbol{\Omega}_{+-}(\sigma)=\int d \sigma^{\prime} \Omega_{+-}\left(\sigma, \sigma^{\prime}\right) .
$$

This result has an important consequence for time evolution of the operator $R$ defined as

$$
R=\int d \sigma\left(c^{+} G_{+}+c^{-} G_{-}+c^{+} \partial_{\sigma} c^{+} \beta_{+}-c^{-} \partial_{\sigma} c^{-} \beta_{-}\right)
$$

since using (3.23) we easily determine that $R$ is conserved up the BRST invariant term

$$
\begin{aligned}
\partial_{\tau} R & =\{Q, \mathbf{R}\} \\
\mathbf{R} & =\int d \sigma\left[c^{+}(\sigma) \boldsymbol{\Omega}_{+-}(\sigma)+c^{-}(\sigma) \boldsymbol{\Omega}_{-+}(\sigma)\right] .
\end{aligned}
$$


Now we consider the Poisson brackets between $G_{ \pm}(\sigma)$ 's. We generally presume that they are non-zero and take the form

$$
\left\{G_{A}(\sigma), G_{B}\left(\sigma^{\prime}\right)\right\}=\mathbf{G}_{A B}\left(\sigma, \sigma^{\prime}\right), \quad \mathbf{G}_{A B}\left(\sigma, \sigma^{\prime}\right)=\mathbf{G}_{B A}\left(\sigma^{\prime}, \sigma\right),
$$

where we used the notation $G_{A}, A= \pm$. For reasons outlined above it is hard to determine the concrete form of the matrix $\mathbf{G}_{A B}$ from the first principles. However let us apply the BRST operator $Q$ on (3.27) for $A=+, B=+$. Then, with the help of generalized Jacobi identity and using (3.18) we obtain

$$
\left\{Q,\left\{G_{+}(\sigma), G_{+}\left(\sigma^{\prime}\right)\right\}\right\}=0
$$

and hence

$$
\left\{Q, \mathbf{G}_{++}\left(\sigma, \sigma^{\prime}\right)\right\}=0
$$

that implies

$$
\mathbf{G}_{++}\left(\sigma, \sigma^{\prime}\right)=\left\{Q, \mathbf{H}_{++}\left(\sigma, \sigma^{\prime}\right)\right\}
$$

for some function $\mathbf{H}_{++}\left(\sigma, \sigma^{\prime}\right)$ of the ghost number -3 . In the same way we obtain

$$
\left\{G_{-}(\sigma), G_{-}\left(\sigma^{\prime}\right)\right\}=\mathbf{G}_{--}\left(\sigma, \sigma^{\prime}\right), \quad \mathbf{G}_{--}=\left\{Q, \mathbf{H}_{--}\left(\sigma, \sigma^{\prime}\right)\right\} .
$$

Finally we apply $Q$ on $\left\{G_{+}(\sigma), G_{-}\left(\sigma^{\prime}\right)\right\}$ and we obtain

$$
\begin{aligned}
& \left\{Q,\left\{G_{+}(\sigma), G_{-}\left(\sigma^{\prime}\right)\right\}\right\}=\left\{T_{-}\left(\sigma^{\prime}\right), G_{+}(\sigma)\right\}+\left\{T_{+}(\sigma), G_{-}\left(\sigma^{\prime}\right)\right\}= \\
& =\Omega_{-+}\left(\sigma^{\prime}, \sigma\right)+\Omega_{+-}\left(\sigma, \sigma^{\prime}\right)=\left\{Q, \mathbf{G}_{+-}\left(\sigma, \sigma^{\prime}\right)\right\} .
\end{aligned}
$$

We see that generally it is not possible to write $\mathbf{G}_{+-}$as $\left\{Q, \mathbf{H}_{+-}\right\}$. We return to this issue below.

Now we are ready to discuss the redefinition of $Q$ as in the previous section. Following the same logic as there we write $Q^{\prime}$ as

$$
Q^{\prime}=Q+\sum_{n=1}^{n} \frac{1}{n !} \overbrace{\{R, \ldots,\{R,\{R, Q\}\}\}}^{n} .
$$

Now we will argue that-as opposite to the case studied in previous section-there are some subtleties with this redefinition when the string is moving in general background. In fact, due to the result (3.26) it is not completely clear that $Q^{\prime}$ is time independent as well. To see this we again consider a quantum mechanics example and calculate

$$
\begin{aligned}
\frac{d Q^{\prime}}{d \tau} & =\frac{d e^{R}}{d \tau} Q e^{-R}+e^{R} \frac{d Q}{d \tau} e^{-R}+e^{R} Q \frac{d e^{-R}}{d \tau}= \\
& =\frac{d e^{R}}{d \tau} Q e^{-R}-e^{R} Q e^{-R} \frac{d e^{R}}{d \tau} e^{-R},
\end{aligned}
$$


where we used $\frac{d Q}{d \tau}=0, \frac{d e^{-R}}{d \tau}=-e^{-R} \frac{d e^{R}}{d \tau} e^{-R}$. We see from the expression above that in order $Q^{\prime}$ to be time-independent we have to demand $\left[R, \partial_{\tau} R\right]=0$. Then $\frac{d e^{R}}{d \tau}=e^{R} \partial_{\tau} R$ and hence

$$
\begin{aligned}
\frac{d Q^{\prime}}{d \tau} & =e^{R} \partial_{\tau} R Q e^{-R}-e^{R} Q \partial_{\tau} R e^{-R}= \\
& =e^{R}([Q, \mathbf{R}] Q-Q[Q, \mathbf{R}]) e^{-R}=e^{R}[[Q, \mathbf{R}], Q] e^{-R}=0,
\end{aligned}
$$

where in the final step we used $\frac{d R}{d \tau}=[Q, \mathbf{R}]$. With the help of the example given above we now return to the classical mechanics. We again presume that

$$
\left\{R, \partial_{\tau} R\right\}=\{R,\{Q, \mathbf{R}\}\}=0
$$

Then we get

$$
\frac{d Q^{\prime}}{d \tau}=e^{R}\left(\left\{\partial_{\tau} R, Q\right\}\right) e^{-R}=e^{R}(\{\{Q, \mathbf{R}\}, Q\}) e^{-R}=0
$$

and hence we obtain that $Q^{\prime}$ is conserved as well. Let us now calculate explicit form of $Q^{\prime}$. It is easy to see that

$$
\begin{aligned}
\{R, Q\} & =\int d \sigma\left(c^{+}\left(T_{+}-\tilde{\beta}^{+} \partial_{\sigma} \tilde{\gamma}^{+}-\partial_{\sigma}\left(\tilde{\gamma}^{+} \tilde{\beta}_{+}\right)+b_{+} \partial_{\sigma} c^{+}\right)+\right. \\
& \left.+c^{-}\left(T_{-}+\tilde{\beta}_{-} \partial_{\sigma} \tilde{\gamma}^{-}+\partial_{\sigma}\left(\tilde{\gamma}^{-} \tilde{\beta}_{-}\right)-b_{-} \partial_{\sigma} c^{-}\right)+\tilde{\gamma}^{+} G_{+}+\tilde{\gamma}^{-} G_{-}\right) .
\end{aligned}
$$

We see that this form coincides with the form of the BRST operator $Q^{\prime}$ derived in previous section. Let us then calculate the second Poisson bracket $\{R,\{R, Q\}\}$. It is clear that the difference with respect to the calculation presented in previous section comes from the possible non-trivial form of the Poisson brackets $\left\{T_{ \pm}, G_{\mp}\right\}$ and $\left\{G_{A}, G_{B}\right\}$. Then after some calculations we obtain

$$
\begin{aligned}
& \{R,\{R, Q\}\}=\{Q, \Sigma\}+ \\
+ & \int d \sigma d \sigma^{\prime}\left[\left(c^{+}(\sigma) \tilde{\gamma}^{-}\left(\sigma^{\prime}\right)+c^{-}(\sigma) \tilde{\gamma}^{+}\left(\sigma^{\prime}\right)\right) \mathbf{G}_{-+}\left(\sigma, \sigma^{\prime}\right)\right],
\end{aligned}
$$

where

$$
\begin{aligned}
& \Sigma=\int d \sigma d \sigma^{\prime} c^{-}(\sigma) c^{+}\left(\sigma^{\prime}\right)\left[\Omega_{+-}\left(\sigma^{\prime}, \sigma\right)-\Omega_{-+}\left(\sigma, \sigma^{\prime}\right)+\right. \\
& \left.+c^{+}(\sigma) \tilde{\gamma}^{+}\left(\sigma^{\prime}\right) \mathbf{H}_{++}\left(\sigma, \sigma^{\prime}\right)+c^{-}(\sigma) \tilde{\gamma}^{-}\left(\sigma^{\prime}\right) \mathbf{H}_{--}\left(\sigma, \sigma^{\prime}\right)\right] .
\end{aligned}
$$

On the other hand, as we argued above, in order to find time-independent BRST operator $Q^{\prime}$ the operator $R$ should obey the relation (3.36). Let us presume that $\partial_{\tau} R=0$ so that

$$
\Omega_{-+}=\Omega_{+-}=0
$$


Then (3.32) implies

$$
\mathbf{G}_{+-}\left(\sigma, \sigma^{\prime}\right)=\left\{Q, \mathbf{H}_{+-}\left(\sigma, \sigma^{\prime}\right)\right\}
$$

and consequently

$$
\{R,\{R, Q\}\}=\left\{Q, \Sigma^{\prime}\right\}
$$

where now

$$
\begin{aligned}
\Sigma^{\prime} & =\int d \sigma d \sigma^{\prime}\left[c^{+}(\sigma) \tilde{\gamma}^{+}\left(\sigma^{\prime}\right) \mathbf{H}_{++}\left(\sigma, \sigma^{\prime}\right)+c^{-}(\sigma) \tilde{\gamma}^{-}\left(\sigma^{\prime}\right) \mathbf{H}_{--}\left(\sigma, \sigma^{\prime}\right)+\right. \\
& \left.+\left(c^{+}(\sigma) \tilde{\gamma}^{-}\left(\sigma^{\prime}\right)+c^{-}(\sigma) \tilde{\gamma}^{+}\left(\sigma^{\prime}\right)\right) \mathbf{H}_{-+}\left(\sigma, \sigma^{\prime}\right)\right]
\end{aligned}
$$

These results imply that the new BRST operator $Q^{\prime}$ contains additional terms as opposite to the BRST operator in flat space-time. This is a natural consequence of the form of the Poisson brackets (3.22) and (3.27). In fact, if the new BRST operator $Q^{\prime}$ is interpreted as the standard BRST operator that contains the first-class constraints only the fact that the Poisson brackets (3.22), (3.27) are non-trivial implies that there are additional constraints that should be taken into account. Moreover, the new form of the BRST operator is not the convention-looking one that is constructed from the first-class constraints only and with corresponding structure constants. In fact, in order to find such a form of the BRST operator we have to presume that all functions $\mathbf{G}_{A B}$ vanish. In this case the new BRST operator takes the same form as the BRST operator in flat space time with difference that $T_{ \pm}$and $G_{ \pm}$are defined for pure spinor string in general background. In other words on condition given above we derive conventional-looking BRST operator in general background that is constructed from the first-class constraints only. This result then opens an interesting possibility to study the classical solution of the pure spinor string in general background since the new BRST operator contains Virasoro constraints that are crucial for correct physical interpretations of these solutions.

\section{Conclusion}

In this section we give a brief summary of our paper. We formulated the pure spinor BRST charge redefinition in the classical manner in order to be able to generalize this to the case of pure spinor string in general background. Then we developed the Hamiltonian formalism for pure spinor string in general background and we found Virasoro constraints. Then we analyzed the general structure of the Poisson brackets and discussed conditions under which the classical redefinition of the BRST charge can be performed.

The motivation for this calculation was to see how Virasoro constraints can emerge from the pure spinor string in general background. In fact, it seems to be rather difficult to study the classical equations of motion for pure spinor string without imposing Virasoro constraints. The reason why we are interested in the study of classical solutions of pure spinor string is following. It is well known that the classical description of the GreenSchwarz superstring in $A d S_{5} \times S^{5}{ }^{10}$ gives very interesting results and predictions. Then

\footnotetext{
${ }^{10}$ For review, see 49, 50].
} 
it would be certainly very interesting to give a covariant form of this analysis using pure spinor formulation of superstring. Then due to the lack of quantum mechanical formulation of pure spinor conformal field theory in this background we wanted to perform classical analysis of pure spinor string in $A d S_{5} \times S^{5}$ as well. However it turned out that the fact that Virasoro constraints are "hidden" in the original pure spinor formulation makes the classical analysis rather obscure. On the other hand we hope that the formulation of the pure spinor theory in $A d S_{5} \times S^{5}$ based on new BRST operator $Q^{\prime}$ could be useful for description of the classical dynamics of the pure spinor string. We currently study this problem and we hope to report about new results in future.

\section{Appendix: Classical Poisson brackets}

In this Appendix we collect some classical Poisson brackets between fundamental modes for pure spinor string in flat background. To begin with we define graded Poisson bracket. Let as consider extended phase space that is spanned with canonical pairs $X^{M}, \Pi_{M}$ with Grassman parity $|M|$. Then the graded Poisson bracket is defined as

$$
\{F, G\}=(-1)^{|F||M|}\left[\frac{\partial^{L} F}{\partial X^{M}} \frac{\partial^{L} G}{\partial \Pi_{M}}-(-1)^{|M|} \frac{\partial^{L} F}{\partial \Pi_{M}} \frac{\partial^{L} G}{\partial X^{M}}\right],
$$

where superscript $L$ on partial derivative means partial left derivative and where the relation between left and right derivative can be found as follows. Let $F$ is function of Grassmann parity $|F|$ defined on superspace labeled with $X^{M}$. Since $d F(Z)=d X^{M} \partial_{M}^{L} F=$

$\partial_{M}^{R} F d X^{M}$ we obtain that left and right derivatives of $F$ are related as $(-1)^{|M||M+F|} \partial_{M}^{L} F=$ $\partial_{M}^{R} F$. In what follows we will consider the derivative from the left only and for that reason we omit the superscript $L$ on the sign of the partial derivative. Note also that the Poisson brackets (5.1) obey relation

$$
\{F, G\}=-(-1)^{|F||G|}\{G, F\}
$$

and generalized Jacobi identity

$$
\{M,\{N, P\}\}+(-1)^{|M||N|+|M||P|}\{N,\{P, M\}\}+(-1)^{|N||P|+|N||M|}\{P,\{M, N\}\}=0 .
$$

Now we return to the pure spinor string in flat background. Using the canonical Poisson brackets (3.7) we easily determine the BRST variations of fundamental modes

$$
\begin{gathered}
\left\{Q_{L}, x^{m}\right\}=i\left(\lambda \gamma^{m} \theta\right), \quad\left\{Q_{L}, p_{m}\right\}=-i \partial_{\sigma}\left(\lambda \gamma^{n} \theta\right) \eta_{m n} \\
\left\{Q_{L}, \theta^{\alpha}\right\}=-\lambda^{\alpha}, \quad\left\{Q_{L}, \lambda^{\alpha}\right\}=0, \quad\left\{Q_{L}, \pi_{\alpha}\right\}=d_{\alpha}
\end{gathered}
$$

while Poisson bracket between $Q_{R}$ and unheated variables all vanish. The same relations can be derived in case of $Q_{R}$ and hatted variables. 
Further, we easily determine

$$
\begin{aligned}
\left\{d_{\alpha}(\sigma), d_{\beta}\left(\sigma^{\prime}\right)\right\} & =2 i \gamma_{\alpha \beta}^{m}\left(p_{m}-\partial_{\sigma} x^{n} \eta_{n m}+2 i\left(\theta \gamma^{n} \partial_{\sigma} \theta\right) \eta_{n m}\right) \delta\left(\sigma-\sigma^{\prime}\right) \equiv \\
& \equiv 2 i \gamma_{\alpha \beta}^{m} \Pi_{m-} \delta\left(\sigma-\sigma^{\prime}\right), \\
\left\{\hat{d}_{\hat{\alpha}}(\sigma), \hat{d}_{\hat{\beta}}\left(\sigma^{\prime}\right)\right\} & =2 i \gamma_{\hat{\alpha} \hat{\beta}}^{m}\left(p_{m}+\partial_{\sigma} x^{n} \eta_{n m}-2 i\left(\hat{\theta} \gamma^{n} \partial_{\sigma} \hat{\theta}\right) \eta_{n m}\right) \delta\left(\sigma-\sigma^{\prime}\right) \equiv \\
& \equiv 2 i \gamma_{\hat{\alpha} \hat{\beta}}^{m} \Pi_{+m} \delta\left(\sigma-\sigma^{\prime}\right), \\
\left\{\hat{d}_{\hat{\alpha}}(\sigma), d_{\beta}\left(\sigma^{\prime}\right)\right\} & =0 .
\end{aligned}
$$

Further, we have

$$
\begin{aligned}
\left\{d_{\alpha}(\sigma), \Pi_{m-}\left(\sigma^{\prime}\right)\right\} & =-4 i\left(\gamma^{n} \partial_{\sigma} \theta\right)_{\alpha} \eta_{n m} \delta\left(\sigma-\sigma^{\prime}\right) \\
\left\{\hat{d}_{\hat{\alpha}}(\sigma), \Pi_{m+}\left(\sigma^{\prime}\right)\right\} & =4 i\left(\gamma^{n} \partial_{\sigma} \hat{\theta}\right)_{\hat{\alpha}} \eta_{n m} \delta\left(\sigma-\sigma^{\prime}\right) \\
\left\{d_{\alpha}(\sigma), \theta^{\beta}\left(\sigma^{\prime}\right)\right\} & =-\delta_{\alpha}^{\beta} \delta\left(\sigma-\sigma^{\prime}\right), \\
\left\{\hat{d}_{\hat{\alpha}}(\sigma), \hat{\theta}^{\hat{\alpha}}\left(\sigma^{\prime}\right)\right\} & =-\delta_{\hat{\alpha}}^{\hat{\beta}} \delta\left(\sigma-\sigma^{\prime}\right) \\
\left\{\Pi_{-m}(\sigma), \Pi_{-n}\left(\sigma^{\prime}\right)\right\} & =-2 \partial_{\sigma} \delta\left(\sigma-\sigma^{\prime}\right) \eta_{n m}, \\
\left\{\Pi_{+m}(\sigma), \Pi_{+n}\left(\sigma^{\prime}\right)\right\} & =2 \partial_{\sigma} \delta\left(\sigma-\sigma^{\prime}\right) \eta_{m n} \\
\left\{\Pi_{-m}(\sigma), \Pi_{n+}\left(\sigma^{\prime}\right)\right\} & =0
\end{aligned}
$$

With the help of these Poisson brackets we obtain

$$
\begin{aligned}
& \left\{Q_{L}, Q_{L}\right\}=2 i \int d \sigma \lambda^{\alpha} \lambda^{\beta} \gamma_{\alpha \beta}^{m} \Pi_{m-} \\
& \left\{Q_{R}, Q_{R}\right\}=2 i \int d \sigma \hat{\lambda}^{\hat{\alpha}} \hat{\lambda}^{\hat{\beta}} \gamma_{\hat{\alpha} \hat{\beta}}^{m} \Pi_{+m}
\end{aligned}
$$

Further, using the Poisson brackets given above we easily get

$$
\begin{aligned}
\left\{Q, \Pi_{-}^{m}\right\} & =-4 i\left(\lambda \gamma^{m} \partial_{\sigma} \theta\right), \quad\left\{Q, \Pi_{+}^{m}\right\}=4 i\left(\hat{\lambda} \gamma^{m} \partial_{\sigma} \hat{\theta}\right) \\
\left\{Q, d_{\alpha}\right\} & =2 i\left(\lambda \gamma^{m}\right)_{\alpha} \Pi_{m-}, \quad\left\{Q, \hat{d}_{\hat{\alpha}}\right\}=2 i\left(\hat{\lambda} \gamma^{m}\right)_{\hat{\alpha}} \Pi_{m+} .
\end{aligned}
$$

Now we determine the Poisson brackets between $Q$ and ghost variables $N, J$ where

$$
\begin{aligned}
& N_{m n}=\frac{1}{2} \pi_{\alpha}\left(\gamma_{m n}\right)_{\beta}^{\alpha} \lambda^{\beta}, \quad J=\pi_{\alpha} \lambda^{\alpha}, \\
& \hat{N}_{m n}=\frac{1}{2} \hat{\pi}_{\hat{\alpha}}\left(\gamma_{m n}\right)_{\hat{\beta}}^{\hat{\alpha}} \hat{\lambda}^{\hat{\beta}}, \quad \hat{J}=\hat{\pi}_{\hat{\alpha}} \hat{\lambda}^{\hat{\alpha}} .
\end{aligned}
$$

Using the free Poisson brackets defined above we easily get

$$
\left\{Q, N_{m n}\right\}=\frac{1}{2}\left(\lambda \gamma_{m n} d\right),\{Q, J\}=d \lambda,
$$




$$
\left\{Q, \hat{N}_{m n}\right\}=\frac{1}{2}\left(\hat{\lambda} \gamma_{m n} \hat{d}\right),\{Q, J\}=\hat{d} \hat{\lambda}
$$

Finally it is also useful to know the Poisson bracket between $N_{m n}$ 's

$$
\left\{N_{m n}(\sigma), N_{k l}\left(\sigma^{\prime}\right)\right\}=\left(\eta_{k n} N_{m l}-N_{m k} \eta_{n l}-\eta_{m k} N_{n l}+\eta_{l m} N_{n k}\right) \delta\left(\sigma-\sigma^{\prime}\right) .
$$

\section{Acknowledgement}

This work was supported by the Czech Ministry of Education under Contract No. MSM 0021622409. 


\section{References}

[1] N. Berkovits, "Super-Poincare covariant quantization of the superstring," JHEP 0004, 018 (2000) [arXiv:hep-th/0001035].

[2] N. Berkovits, "ICTP lectures on covariant quantization of the superstring," arXiv:hep-th/0209059.

[3] P. A. Grassi, " $N=2$ superparticles, $R R$ fields and noncommutative structures of (super)-spacetime," arXiv:hep-th/0511015.

[4] P. A. Grassi, G. Policastro and P. van Nieuwenhuizen, "An introduction to the covariant quantization of superstrings," Class. Quant. Grav. 20 (2003) S395 [arXiv:hep-th/0302147].

[5] P. A. Grassi, G. Policastro and P. van Nieuwenhuizen, "Yang-Mills theory as an illustration of the covariant quantization of superstrings," arXiv:hep-th/0211095.

[6] N. A. Nekrasov, "Lectures on curved beta-gamma systems, pure spinors, and anomalies," arXiv:hep-th/0511008.

[7] N. Berkovits, "Multiloop amplitudes and vanishing theorems using the pure spinor formalism for the superstring," JHEP 0409 (2004) 047 [arXiv:hep-th/0406055].

[8] N. Berkovits and N. Nekrasov, "Multiloop superstring amplitudes from non-minimal pure spinor formalism," JHEP 0612 (2006) 029 [arXiv:hep-th/0609012].

[9] C. R. Mafra, "Pure Spinor Superspace Identities for Massless Four-point Kinematic Factors," arXiv:0801.0580 [hep-th].

[10] N. Berkovits and O. Chandia, "Superstring vertex operators in an AdS(5) x S(5) background," Nucl. Phys. B 596, 185 (2001) [arXiv:hep-th/0009168].

[11] N. Berkovits, "Conformal field theory for the superstring in a Ramond-Ramond plane wave background," JHEP 0204 (2002) 037 [arXiv:hep-th/0203248].

[12] N. Berkovits, "Quantum consistency of the superstring in AdS(5) x $S^{* * 5}$ background," JHEP 0503, 041 (2005) [arXiv:hep-th/0411170].

[13] A. Mikhailov and S. Schafer-Nameki, "Algebra of transfer-matrices and Yang-Baxter equations on the string worldsheet in $A d S(5) \times S(5)$," arXiv:0712.4278 [hep-th].

[14] A. Mikhailov and S. Schafer-Nameki, "Perturbative study of the transfer matrix on the string worldsheet in $A d S(5) x S(5)$," arXiv:0706.1525 [hep-th].

[15] N. Berkovits and C. Vafa, "Towards a Worldsheet Derivation of the Maldacena Conjecture," arXiv:0711.1799 [hep-th].

[16] N. Berkovits, "A New Limit of the AdS $S_{5} x S^{5}$ Sigma Model," JHEP 0708 (2007) 011 [arXiv:hep-th/0703282].

[17] P. A. Grassi and J. Kluson, "Pure spinor strings in TsT deformed background," JHEP 0703 (2007) 033 [arXiv:hep-th/0611151].

[18] V. G. M. Puletti, "Operator product expansion for pure spinor superstring on AdS(5) $x$ $S^{* * 5}$," JHEP 0610 (2006) 057 [arXiv:hep-th/0607076].

[19] M. Bianchi and J. Kluson, "Current algebra of the pure spinor superstring in $A d S(5) x S(5)$," JHEP 0608 (2006) 030 [arXiv:hep-th/0606188]. 
[20] J. Kluson, "Note about classical dynamics of pure spinor string on AdS(5) x $S^{* * 5}$

background," Eur. Phys. J. C 50 (2007) 1019 [arXiv:hep-th/0603228].

[21] N. Berkovits, "BRST cohomology and nonlocal conserved charges," JHEP 0502 (2005) 060 [arXiv:hep-th/0409159].

[22] B. C. Vallilo, "Flat currents in the classical AdS(5) $x S^{* *} 5$ pure spinor superstring," JHEP 0403 (2004) 037 [arXiv:hep-th/0307018].

[23] B. C. Vallilo, "One loop conformal invariance of the superstring in an AdS(5) $x S(5)$ background," JHEP 0212 (2002) 042 [arXiv:hep-th/0210064].

[24] N. Berkovits, "Relating the RNS and pure spinor formalisms for the superstring," JHEP 0108, 026 (2001) [arXiv:hep-th/0104247].

[25] I. Oda and M. Tonin, "On the Berkovits covariant quantization of GS superstring," Phys. Lett. B 520 (2001) 398 [arXiv:hep-th/0109051].

[26] P. A. Grassi and P. van Nieuwenhuizen, " $N=4$ superconformal symmetry for the covariant quantum superstring," Phys. Lett. B 610 (2005) 129 [arXiv:hep-th/0408007].

[27] P. A. Grassi, G. Policastro and P. van Nieuwenhuizen, "Superstrings and WZNW models," arXiv:hep-th/0402122.

[28] S. Guttenberg, J. Knapp and M. Kreuzer, "On the covariant quantization of type II superstrings," JHEP 0406 (2004) 030 [arXiv:hep-th/0405007].

[29] P. A. Grassi, G. Policastro and P. van Nieuwenhuizen, "The quantum superstring as a WZNW model," Nucl. Phys. B 676 (2004) 43 [arXiv:hep-th/0307056].

[30] I. Oda and M. Tonin, "Y-formalism in pure spinor quantization of superstrings," Nucl. Phys. B 727 (2005) 176 [arXiv:hep-th/0505277].

[31] Y. Aisaka and Y. Kazama, "Origin of pure spinor superstring," JHEP 0505 (2005) 046 [arXiv:hep-th/0502208].

[32] M. Chesterman, "On the cohomology and inner products of the Berkovits superparticle and superstring," Nucl. Phys. B 703 (2004) 400 [arXiv:hep-th/0404021].

[33] Y. Aisaka and Y. Kazama, "Operator mapping between RNS and extended pure spinor formalisms for superstring," JHEP 0308 (2003) 047 [arXiv:hep-th/0305221].

[34] M. Matone, L. Mazzucato, I. Oda, D. Sorokin and M. Tonin, "The superembedding origin of the Berkovits pure spinor covariant quantization of superstrings," Nucl. Phys. B 639 (2002) 182 [arXiv:hep-th/0206104].

[35] P. A. Grassi, G. Policastro and P. van Nieuwenhuizen, "The covariant quantum superstring and superparticle from their classical actions," Phys. Lett. B 553 (2003) 96 [arXiv:hep-th/0209026].

[36] N. Berkovits, "Pure spinor formalism as an $N=2$ topological string," JHEP 0510 (2005) 089 [arXiv:hep-th/0509120].

[37] N. Berkovits and D. Z. Marchioro, "Relating the Green-Schwarz and pure spinor formalisms for the superstring," JHEP 0501 (2005) 018 [arXiv:hep-th/0412198].

[38] A. Gaona and J. A. Garcia, "BFT embedding of the Green-Schwarz superstring and the pure spinor formalism," JHEP 0509 (2005) 083 [arXiv:hep-th/0507076]. 
[39] J. Hoogeveen and K. Skenderis, "BRST quantization of the pure spinor superstring," JHEP 0711 (2007) 081 [arXiv:0710.2598 [hep-th]].

[40] N. Berkovits, "Explaining the Pure Spinor Formalism for the Superstring," JHEP 0801 (2008) 065 [arXiv:0712.0324 [hep-th]].

[41] N. Berkovits and P. S. Howe, 'Ten-dimensional supergravity constraints from the pure spinor formalism for the superstring," Nucl. Phys. B 635, 75 (2002) [arXiv:hep-th/0112160].

[42] R. Benichou, G. Policastro and J. Troost, "T-duality in Ramond-Ramond backgrounds," arXiv:0801.1785 [hep-th].

[43] O. Chandia and M. Tonin, "BRST Anomaly and Superspace Constraints of the Pure Spinor Heterotic String in a Curved Background," JHEP 0709 (2007) 016 [arXiv:0707.0654 [hep-th]].

[44] I. Adam, A. Dekel, L. Mazzucato and Y. Oz, "Integrability of type II superstrings on Ramond-Ramond backgrounds in various dimensions," JHEP 0706 (2007) 085 [arXiv:hep-th/0702083].

[45] O. Chandia, "A note on the classical BRST symmetry of the pure spinor string in a curved background," JHEP 0607 (2006) 019 [arXiv:hep-th/0604115].

[46] O. Chandia and B. C. Vallilo, "Conformal invariance of the pure spinor superstring in a curved background," JHEP 0404 (2004) 041 [arXiv:hep-th/0401226].

[47] N. Berkovits, "Covariant quantization of the supermembrane,” JHEP 0209 (2002) 051 [arXiv:hep-th/0201151].

[48] J. A. Shapiro and C. C. Taylor, "THE SPACE-TIME SUPERSYMMETRIC FORMULATION OF THE STRING," Phys. Rept. 191 (1990) 221.

[49] A. A. Tseytlin, "Spinning strings and AdS/CFT duality," arXiv:hep-th/0311139.

[50] J. Plefka, "Spinning strings and integrable spin chains in the AdS/CFT correspondence," Living Rev. Rel. 8 (2005) 9 [arXiv:hep-th/0507136]. 